\title{
Evaluación de aceite de Jatropha gossypiifolia como estabilizante de asfaltenos en una muestra de petróleo a nivel de laboratorio
}

\author{
Tomás Darío Marín Velásquez* \\ Universidad de Oriente, Departamento de Ingeniería de Petróleo, Laboratorio de Procesamiento de Hidrocarburos, \\ Maturín, Monagas, Venezuela. \\ E-mail: tmarin@protonmail.com
}

\begin{abstract}
Resumen
Los asfaltenos son sólidos poliaromáticos condensados que se encuentran presentes en el petróleo formando suspensiones coloidales, en un sistema en termodinámico que en algunos casos tiende a ser inestable produciendo floculación y agregación. En la industria petrolera, se usan compuestos químicos dispersantes para minimizar la floculación de asfaltenos, para evitar problemas de taponamiento en tuberías y equipos de producción, lo que representa una inversión importante ya que los mismos se formulan con resinas y aceites sintéticos, además de solventes costosos. En la investigación se evalúa el uso del aceite de la Jatropha gossypifolia como componente activo estabilizante de asfaltenos, mezclado con gasoil como solvente en ensayos realizados a nivel de laboratorio. Se evaluaron cinco disoluciones de aceite en gasoil (10 a 50\%). El procedimiento consistió en observar en un microscopio óptico los agregados de asfaltenos al aplicar n-heptano a una muestra de petróleo crudo del Estado Monagas, Venezuela, siendo el volumen de n-heptano utilizado el umbral de floculación. Luego se aplicaron volúmenes sucesivos de cada disolución hasta lograr la dispersión de los agregados, siendo este volumen el punto de dispersión. Adicionalmente, se usó xileno como dispersante patrón para comparar el desempeño de las disoluciones. Los ensayos se realizaron a dos temperaturas 25 y $40^{\circ} \mathrm{C}$. Se utilizó un diseño estadístico factorial con factores experimentales disolución y temperatura y variable dependiente índice de inestabilidad, con análisis ANOVA y test HSD de Tukey con $\alpha=0,05$. Con la aplicación de las disoluciones se logró una mayor dispersión de los agregados con eficiencias mayores a 94\% respecto a la dispersión obtenida con el xileno, siendo la disolución con $40 \%$ aceite la más eficiente. Los resultados estadísticos mostraron que la estabilidad es dependiente de la disolución utilizada e independiente de la temperatura de la prueba con un nivel de confianza de $95 \%$.
\end{abstract}

Palabras clave: Asfaltenos, dispersión, estabilidad, floculación, Jatropha gossypiifolia, petróleo crudo.

\section{Evaluation of Jatropha gossypiifolia oil as a asphaltenes stabilizer in a petroleum sample at the laboratory level}

\begin{abstract}
Asphaltenes are condensed polyaromatic solids that are present in oil forming colloidal suspensions in a thermodynamic system that in some cases tends to be unstable producing flocculation and aggregation. In the oil industry, dispersant chemical compounds are used to minimize the flocculation of asphaltenes to avoid clogging problems in pipes and production equipment, which represent an important investment since they are formulated with synthetic resins and oils, in addition to expensive solvents. This research evaluates the use of Jatropha gossypifolia oil as an active stabilizing component of asphaltenes, mixed with diesel as a solvent in laboratory level tests. Five dilutions of oil in diesel (10 to 50\%) were evaluated. The procedure consisted of observing the asphaltene aggregates under an optical microscope when applying n-heptane to a crude oil sample from Monagas State, Venezuela, the volume of $n$-heptane used being the flocculation onset. Successive volumes of each dilution were then applied until the dispersion of the aggregates was achieved, being this volume the point of dispersion. In addition, xylene was used as a standard dispersant to compare the performance of the dissolutions. The tests were performed at two temperatures of 25 and $40{ }^{\circ} \mathrm{C}$. A factorial statistical design was used with experimental factors dissolution and temperature and dependent variable instability index, with ANOVA analysis and Tukey HSD test with $\alpha=0.05$. With the application of the solutions, a greater dispersion of the aggregates was achieved with efficiencies greater than $94 \%$ with respect to the dispersion obtained with xylene, being the solution with $40 \%$ oil the most efficient. The statistical results showed that stability is dependent on the solution used and independent of the test temperature with a $95 \%$ confidence level.
\end{abstract}

Keywords: Asphaltenes, dispersion, stability, flocculation, Jatropha gossypiifolia, crude oil.

Cita: Marín Velásquez, T. D. (2020). Evaluación de aciete de Jatropha gossypiifolia como estabilizante de asfaltenos en una muestra de petróleo a nivel de laboratorio. Revista Fuentes: El reventón energético, 18(1), 17-29. 


\section{Introducción}

El petróleo es un recurso no renovable que está constituido en su mayoría por hidrocarburos. Entre éstos, existen sólidos de alto peso molecular y compuestos de estructura compleja, con diferentes proporciones de nitrógeno, azufre y oxígeno, conocidas conjuntamente por el nombre genérico de asfaltenos (Pineda-Flores \& Mesta-Howard, 2001). Un esquema simple que se ha utilizado durante décadas para separar los componentes del petróleo crudo en fracciones de saturados, aromáticos, resinas y asfaltenos dependiendo de sus solubilidades, se conoce como fraccionamiento SARA (Kharrat et al. 2007; León, 2011; León, González \& Arango, 2007).

A pesar de la diversidad de criterios que se adoptan al definir los asfaltenos, se ha llegado a un consenso al catalogarlos como la fracción del petróleo soluble en aromáticos como tolueno, xileno o benceno e insoluble en n-alcanos como pentano, hexan o heptano (Spiecker, Gawrys \& Kilpatrick, 2003). Según Goual (2012), la definición de la clase de solubilidad de los asfaltenos genera una amplia distribución de las estructuras moleculares. Estas moléculas polidispersas consisten principalmente en aromáticos polinucleares (PNA) con diferentes proporciones de alifáticos y alicíclicos y pequeñas cantidades de heteroátomos (como oxígeno, nitrógeno, azufre) y metales pesados, como vanadio y níquel, que se encuentran en estructuras de porfirina (Romero, Feitosa, Do Carmo \& De Sant'Ana, 2018).

Los asfaltenos por ser los componentes más pesados del petróleo y por encontrarse en un estado de pseudoestabilidad, bajo ciertas condiciones desfavorables de presión, temperatura, composición química y tasa de corte, se precipitan afectando de manera negativa la recuperación de petróleo (Sulaimon, De Castro \& Vatsa, 2019; Sepúlveda, Bonilla \& Medina, 2010; Figueira, Simão, Soares \& Lucas, 2017). La teoría más aceptada para definir el comportamiento de los asfaltenos en el petróleo es la teoría coloidal, en la cual los asfaltenos existen en el petróleo como partículas sólidas en suspensión coloidal estabilizada por resinas adsorbidas sobre su superficie (Akbarzadeh et al., 2007; Mansoori, 2009; Chavez, Campo, Morales \& Carrillo, 2011).

Al estar los asfaltenos en suspensión dentro del petróleo, su estabilidad depende de las condiciones termodinámicas a las que está sometido éste (Presión, Temperatura y Composición) por lo tanto, variaciones en estas condiciones pueden generar rompimiento del sistema coloidal, formación de agregados y separación de los mismos de la fase líquida, produciendo depósitos orgánicos (Campen, Moorhouse \& Wong, 2019; Safaie $\&$ Naza, 2014). Para mantener la estabilidad se utilizan productos químicos dispersantes, formulados a base de resinas sintéticas y solventes aromáticos (Saeedi \& Hasan, 2017; Pereira et al., 2011). Los componentes activos de los productos dispersantes de asfaltenos son las resinas, las cuales son consideradas surfactantes y cuyo principio de acción depende del tipo específico de la misma, de su composición y grupos funcionales que posean (Madh, Kharrat \& Hamoule, 2017; León, Quintero, Cervantes \& Rangel, 2012; Sánchez, Sánchez, González \& Ramírez, 2017).

El uso de compuestos surfactantes como dispersantes de asfaltenos, ha sido estudiado ampliamente en búsqueda de mejores resultados en la estabilización y prevención de los problemas de taponamiento, el cual se debe al fenómeno de floculación y precipitación, formando depósitos orgánicos sólidos formados además por resinas y ceras parafínicas (Lim, Go, Nho \& Lee, 2018; Mehrotra \& Bidmus, 2004; García et al, 2010). En su investigación Rezaee et al. (2013), se enfocaron en el estudio de un surfactante iónico ácido (3-(2-carboxibenzoil)-1-metil-1H-imidazolcloruro de 3-ion) como dispersante de asfaltenos. Ghaffar et al. (2013) también estudiaron un surfactante polimérico oxigenado tipo dodecilfenol formaldehído-b-poli (oxipropileno). En ambos casos se estudiaron compuestos sintéticos derivados de hidrocarburos del petróleo.

Además del estudio de diferentes compuestos sintéticos, las metodologías y protocolos de ensayo para la determinación de la eficiencia de productos dispersantes de asfaltenos ha sido motivo de estudio en diversas investigaciones. En tal sentido, Andersen (1999), menciona que existen diferentes métodos para determinar el umbral de floculación y la eficiencia de productos dispersantes, que se basan en la titulación con un no disolvente (floculante) hasta que se observa una fase sólida, entre las que se encuentran la prueba de manchas de Oliensis cuyo principio es el examen de gotas aplicadas sobre papel de filtro, examen microscópico de soluciones, transmisión óptica y dispersión de la luz por partículas, mediciones de conductividad, viscosimetría, espectroscopia de fluorescencia, análisis de tamaño de partículas y análisis de transferencia de calor.

Debido a los gastos que ocasiona el uso de productos cuyos componentes activos son formulados sintéticamente y el riesgo ambiental que los mismos representan, se han venido realizando estudios con 
aceites y resinas de origen vegetal, como el aceite de coco (Bello, Manzano \& Marín, 2015), el aceite de avellana (Safaie \& Naza, 2014), aceite de Jatropha curcas (Marín, Marcano \& Febres, 2016) y el aceite obtenido de la cáscara del Anacardiun occidentale (Afra et al., 2018). En todas las investigaciones se han demostrado la aplicabilidad de los aceites vegetales y sus derivados como inhibidores o dispersantes de asfaltenos con eficiencias mayores a $80 \%$. Por lo anterior, se propuso la siguiente investigación utilizando el aceite de Jatropha gossypiifolia como dispersante de asfaltenos, en búsqueda de una nueva alternativa de tratamiento para el petróleo producido en el Norte del Estado Monagas, Venezuela, con el uso de un aceite no comestible y que puede ser de fácil obtención, debido a que proviene de una especie vegetal que crece de forma silvestre.

La Jatropha gossypiifolia es una planta de la familia Euphorbiaceae, originaria de América y que se encuentra distribuida en países de clima tropical y subtropical de América y África (Félix-Silva et al., 2014). El uso de diferentes partes de la planta ha sido estudiado ampliamente por su potencial medicinal (Oyedeji et al., 2018; Yaro et al., 2018; Islam, Islam \& Hossain, 2017; De Almeida et al., 2016), sin embargo, existen pocos estudios de su aplicación como biocombustible. Al ser ésta una especie vegetal perteneciente a la familia Euphorbiaceae, que crece de forma silvestre distribuida entre las regiones tropicales y subtropicales de América, que posee propiedades medicinales, ha sido ampliamente estudiada $\mathrm{y}$ documenta, sobre todo por el uso de los extractos de sus hojas, tallos y raíces para el tratamiento de diversas enfermedades (Wu et al., 2019; Xavier-Santos et al., 2018; De Almeida et al., 2015; Félix-Silva et al., 2014), así como para uso comercial como colorante para el teñido de algodón (Kumar \& Singh, 2012), sin embargo, las propiedades y usos del aceite extraído de las semillas no ha sido tomado muy en cuenta hasta los momentos, más allá de investigaciones encaminadas a establecer su composición. El aceite ha sido poco estudiado, debido a que es más comercial el aceite de Jatropha curcas, por su potencial para producir biocombustible, mayor rendimiento de aceite, mayor distribución geográfica y mejoras genéticas que se le han realizado. El uso de la Jatropha gossypiifolia se ha limitado en estudios previos, a comparación en composición y comportamiento con otras plantas de la especie Jatropha. De lo anterior deriva la importancia de su evaluación como agente dispersante de asfaltenos y su posible uso en la industria petrolera.

\section{Metodología}

\subsection{Obtención de la materia prima}

Para la obtención de la semilla de Jatropha gossypiifolia se realizó colecta manual directamente de plantas, ubicadas en la zona Este del Estado Monagas, posteriormente, se sometieron a un secado al aire libre sin que incidiera directamente los rayos solares durante tres días, luego, se trituraron en un mortero y se conservó a temperatura ambiente. La muestra de petróleo crudo fue obtenida del Campo el Furrial ubicado en el área Norte del Estado Monagas a $30 \mathrm{~km}$ de la ciudad de Maturín. Se escogió una muestra de esta zona pues en la misma se producen petróleos con problemas de precipitación de asfaltenos (Alayón, 2004).

\subsection{Obtención del Aceite de Jatropha gossypiifolia}

El aceite de Jatropha gossypiifolia fue obtenido mediante extracción sólido líquido utilizando con extractor el n-hexano y mediante un equipo de extracción Sohxlet. Se utilizaron $100 \mathrm{~g}$ de semilla triturada y $800 \mathrm{~mL}$ de n-hexano, para lo que se contó con cuatro equipos de extracción en los que se colocaron 25 g de semilla y $200 \mathrm{~mL}$ de n-hexano por equipo. La extracción se llevó a cabo por 4 hrs. El aceite más el solvente se llevó a un rotoevaporador en el cual se separó el solvente a una temperatura de $68^{\circ} \mathrm{C}$ y se concentró el aceite extraído.

\subsection{Caracterización de la muestra de petróleo crudo}

La muestra se caracterizó de acuerdo a procedimientos establecidos en las normas de la American Society for Testing and Materials (ASTM) y las propiedades determinadas fueron, la gravedad API por el método del hidrómetro (ASTM D287, 2012), Viscosidad dinámica mediante un viscosímetro Brookfield (ASTM D2196, 2010) y Análisis SARA por cromatografía en sílica gel (ASTM D2007, 2011).

\subsection{Caracterización del aceite de Jatropha gossypiifolia y las disoluciones del mismo en gasoil}

Se determinó para el aceite de Jatropha gossypiifolia, el rendimiento en porcentaje másico, mediante la ecuación 1 :

$$
R=\frac{\text { masa de aceite }(g)}{\text { masa de semilla }(g)}=x 100
$$


La densidad se obtuvo mediante el método del picnómetro (ASTM D891, 2008) y la viscosidad cinemática mediante tubos capilares calibrados (ASTM D445, 2011). Se prepararon cinco disoluciones con 10, $20,30,40$ y $50 \%$ de aceite en gasoil, que también fueron caracterizadas con base en los mismos procedimientos aplicados para el aceite puro. No se utilizó el gasoil puro debido a que en investigaciones anteriores con diferentes tipos de petróleo, se demostró que el mismo no ejerce ningún efecto estabilizante sobre los asfaltenos (Cuffaro \& Centeno, 2017).

El diésel como solvente de los aceites se utilizó debido a su naturaleza aromática-alifática, con un contenido de aromáticos entre 37 y 38\% (Salazar, Rodríguez \& Marín, 2017).

\subsection{Obtención del umbral de floculación y punto de dispersión de asfaltenos del petróleo crudo}

Para la obtención del umbral de floculación, se midieron $10 \mathrm{~mL}$ de la muestra de petróleo crudo en un vaso de precipitado de $50 \mathrm{~mL}$, se colocó en el sistema de calentamiento sobre un agitador magnético y se agitó a velocidad constante (Figura 1).

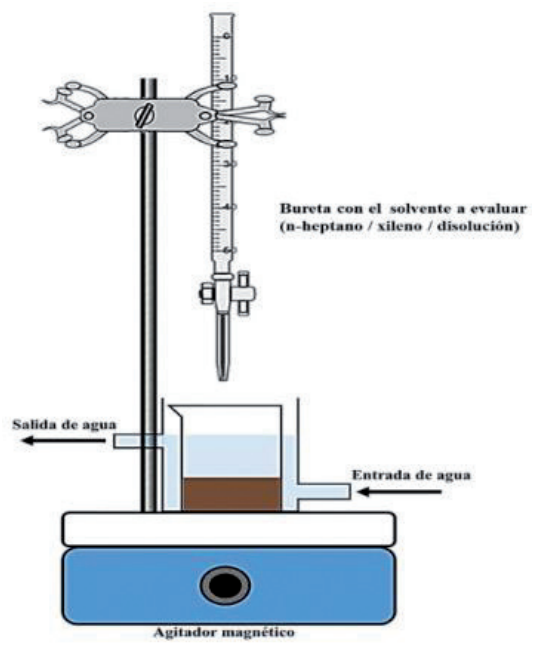

Figura 1. Esquema del sistema de laboratorio utilizado para la medición del INE. Fuente: elaboración propia.

Luego se aplicó n-heptano, en porciones de $0,5 \mathrm{~mL}$ agitando por $5 \mathrm{~min}$. Se tomó una alícuota y se colocó una gota en un portaobjeto para después de $5 \mathrm{~min}$ de reposo, observarla a través de un microscopio óptico marca Optima, Modelo XSZ-207 con objetivo y ocular 10X, hasta observar la presencia de los agregados de asfaltenos (Marín, Marcano \& Febres, 2016). El umbral de floculación (UF) se toma como el volumen de $\mathrm{n}$-heptano en mililitros necesario para obtener agregados visibles.
El punto de dispersión se determinó aplicando un disolvente de asfaltenos aromático (xileno) a las muestras con asfaltenos agregados (obtenidos anteriormente) en porciones de $0,5 \mathrm{~mL}$ agitando por $5 \mathrm{~min}$, colocando una gota en un portaobjeto y observando luego de 5 min de reposo en el microscopio hasta observar la dispersión o disolución de los agregados de asfaltenos. El punto de dispersión (PD) se representa como la cantidad de Xileno aplicado para lograr la dispersión de los agregados (Marín, Marcano \& Febres, 2016). Se calculó la relación entre los volúmenes de dispersante y el volumen de floculante (índice de inestabilidad), mediante la ecuación 2 :

$$
I N E=\frac{P D(m L)}{U F(m L)}
$$

En la figura 1 se muestra el esquema del equipo utilizado para la determinación del INE, el cual consta de una bureta con el solvente a evaluar (n-heptano, xileno o disolución), un sistema de calentamiento conectado a un baño termostático de inmersión y circulación marca Julabo modelo MP, rango de temperatura 20 a $100{ }^{\circ} \mathrm{C}$ y bomba de $10 \mathrm{ml} / \mathrm{min}$ de flujo, un agitador magnético marca Corning modelo PC-220 y la muestra de petróleo dentro de un vaso de precipitado.

La observación del proceso de agregación y dispersión de los asfaltenos se realizó mediante un microscopio óptico, con el que se obtuvieron adicionalmente microfotografías. En la figura 2, se muestran ejemplos de microfotografías tomadas durante los ensayos realizados con la muestra de petróleo en estudio.
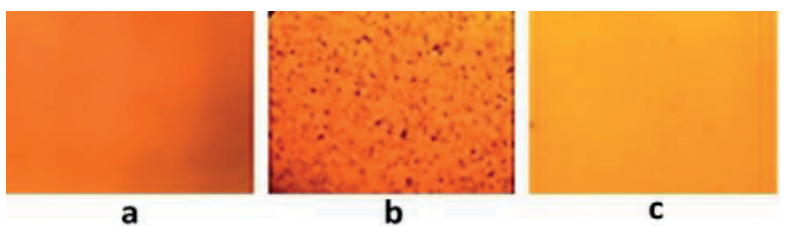

Figura 2. Ejemplos de microfotografías obtenidas. 1a: muestra de petróleo original. 1b: muestra con agregados de asfaltenos en el umbral de floculación. 1c: muestra con asfaltenos dispersos en el punto de dispersión.

\subsection{Determinación de la eficiencia de dispersión de las disoluciones de aceite en gasoil}

Para medir la aplicabilidad del aceite de Jatropha gossypiifolia como agente activo dispersante de asfaltenos, se determinaron los puntos de dispersión aplicando cada una de las disoluciones mediante el procedimiento descrito anteriormente. Se sustituyó en el ensayo el Xileno como dispersante por cada una de las disoluciones. 
La eficiencia de dispersión de cada una de las disoluciones se calculó con base en la INE original utilizando el xileno, tomándose como valor base mediante la ecuación 3 :

$$
\% E f=\frac{\text { INE xileno }- \text { INE disolución }}{\text { INE xilenox }} \times 100
$$

Los ensayos se realizaron a temperatura ambiente $(25$ ${ }^{\circ} \mathrm{C}$ ) y calentando la muestra de petróleo a $40{ }^{\circ} \mathrm{C}$, con la finalidad de observar el posible efecto de la temperatura sobre el desempeño de las disoluciones. Todos los ensayos se realizaron por triplicado.

\subsection{Tratamiento estadístico de los resultados}

Los tratamientos (disoluciones) se identificaron según se muestra en la tabla 1.

Tabla 1. Identificación de los tratamientos utilizados

\begin{tabular}{|c|c|c|}
\hline Disolución & $\begin{array}{c}\text { Aceite de Jatropha } \\
\text { gossypiifolia (\%) }\end{array}$ & Gasoil (\%) \\
\hline A & 10 & 90 \\
\hline B & 20 & 80 \\
\hline C & 30 & 70 \\
\hline D & 40 & 60 \\
\hline E & 50 & 50 \\
\hline
\end{tabular}

Fuente: elaboración propia.

Los resultados de eficiencia fueron analizados mediante un ANOVA Multifactorial, para estudiar los efectos aislados de los factores experimentales disolución y temperatura sobre la variable dependiente INE, así como los efectos de interacción entre estos dos factores utilizando la prueba de Diferencia Significativa Mínima de Tukey (HSD), con un nivel de significancia de 0,05. El diseño experimental fue factorial con dos factores y una variable respuesta. Se utilizó el paquete estadístico Statgraphics Centurion XVI.I.

\section{Resultados}

Luego de la aplicación de los ensayos de laboratorio se obtuvieron las propiedades de la muestra de petróleo crudo. En la tabla 2 se observa que la muestra de petróleo crudo es de grado mediano según su gravedad API, la cual está dentro del rango comprendido entre 22 y 29,9 .

De acuerdo al análisis SARA se observa que los componentes mayoritarios de la muestra de Petróleo son los saturados y los aromáticos, los cuales representan el $88,9 \%$ de su composición, representando las resinas y los asfaltenos el restante $11,1 \%$. De la relación Resina/ Asfaltenos se puede deducir la estabilidad del petróleo, es decir su tendencia producir depósitos de asfaltenos. La relación entre estas facciones es de 2,3 lo que lo ubica en el rango de inestabilidad por ser menor a 15 (Sepúlveda et al., 2010). También, el índice de estabilidad coloidal según Asomaning y Watkinson (2000) es de 0,95 que, también indica inestabilidad por ser mayor a 0,90 según lo establecido por los autores citados.

Tabla 2. Características de la muestra de petróleo.

\begin{tabular}{|l|c|c|}
\hline Propiedad & Unidad & Valor \\
\hline Gravedad API & Adim & 26,5 \\
\hline Viscosidad @ 23 ${ }^{\circ} \mathrm{C}$ & $\mathrm{cP}$ & 41,3 \\
\hline Saturados & $\%$ & 45,3 \\
\hline Aromáticos & $\%$ & 43,6 \\
\hline Resinas & $\%$ & 7,7 \\
\hline Asfaltenos & $\%$ & 3,4 \\
\hline
\end{tabular}

Fuente: elaboración propia.

Las característica del aceite de Jatropha gossypiifolia se muestran en la tabla 3 , donde se observa un rendimiento de $27 \%$, lo que significa que se obtienen $27 \mathrm{~g}$ de aceite $(30,9 \mathrm{~mL})$ por cada $100 \mathrm{~g}$ de semillas procesadas.

Tabla 3. Características del aceite de Jatropha gossypiifolia.

\begin{tabular}{|l|c|c|}
\hline Propiedad & Unidad & Valor \\
\hline Rendimiento & $\%$ & 27,0 \\
\hline Densidad & $\mathrm{g} / \mathrm{mL}$ & 0,88 \\
\hline Viscosidad@ $24{ }^{\circ} \mathrm{C}$ & $\mathrm{cSt}$ & 5,98 \\
\hline
\end{tabular}

Fuente: elaboración propia.

La densidad de $0,88 \mathrm{~g} / \mathrm{mL}$ muestra que el aceite es más liviano que el agua y que la muestra de petróleo, cuya densidad fue de $0,90 \mathrm{~g} / \mathrm{mL}$. También se obtuvo una viscosidad menor a la de la muestra de petróleo, por lo que se tiene un aceite bastante liviano y con buena fluidez. Al comparar con las propiedades de gasoil utilizado, el aceite resultó ser más pesado y viscoso, ya que el gasoil presentó densidad de $0,85 \mathrm{~g} / \mathrm{mL}$ y viscosidad de $5,14 \mathrm{cSt}$.

En la tabla 4, se muestran los resultados obtenidos al someter a la muestra de petróleo a los solventes n-heptano y xileno para determinar el umbral de floculación, el punto de dispersión y el INE originales de la misma.

Tabla 4. Umbrales de floculación, puntos de dispersión e índice de inestabilidad del petróleo.

\begin{tabular}{|l|c|c|c|}
\hline Propiedad & Unidad & Valor a $25^{\circ} \mathbf{C}$ & Valor a $40{ }^{\circ} \mathbf{C}$ \\
\hline UF & $\mathrm{mL}$ & 9,5 & 10,0 \\
\hline $\mathrm{PD}$ & $\mathrm{mL}$ & 3,50 & 5,50 \\
\hline $\mathrm{INE}$ & $\mathrm{mL} / \mathrm{mL}$ & 0,37 & 0,55 \\
\hline
\end{tabular}

Fuente: elaboración propia. 
El valor de índice de inestabilidad refleja la cantidad de dispersante en $\mathrm{mL}$ necesaria para disolver los asfaltenos, por cada $\mathrm{mL}$ de agente floculante aplicado para obtener los agregados de asfaltenos; siendo el petróleo más estable mientras menor sea este índice. Lo anterior indica que la muestra de petróleo crudo al ser analizada a temperatura de $25^{\circ} \mathrm{C}$ fue más estable que al ser analizada a $40^{\circ} \mathrm{C}$, lo que sugiere que este petróleo en particular tiende a desestabilizarse con la temperatura. El efecto de la temperatura no sigue un patrón común, sin embargo autores como Maqbool et al. (2011) también demostraron que a temperaturas más altas el tiempo de inicio de precipitación para los asfaltenos es más corto, considerando que el efecto del aumento de la temperatura influye directamente en las propiedades como viscosidad y la densidad, además de la expansión, oxidación y evaporación de hidrocarburos, son factores determinantes en el comportamiento de la estabilidad.

En la tabla 5 se observa como la viscosidad es el parámetro más afectado por la mezcla entre al aceite de Jatropha gossypiifolia y el gasoil teniendo una tendencia al aumento al ir incrementando la cantidad de aceite en la disolución. La densidad también aumenta pero no de forma continua, sino escalonada.

Tabla 5. Propiedades de las disoluciones de aceite de Jatropha gossypiifolia en gasoil.

\begin{tabular}{|c|c|c|c|}
\hline Disolución & \%Aceite & Densidad g/mL & Viscosidad cSt \\
\hline A & 10 & 0,80 & 5,57 \\
\hline B & 20 & 0,80 & 7,03 \\
\hline C & 30 & 0,81 & 7,95 \\
\hline D & 40 & 0,84 & 8,66 \\
\hline E & 50 & 0,84 & 9,71 \\
\hline
\end{tabular}

Fuente: elaboración propia.

Se observa entonces que de las dos propiedades medidas para las disoluciones, el efecto más importante ocurre en la viscosidad, pues el cambio puede considerarse significativo, ya que el porcentaje de variación fue de $20,29 \%$. En el caso de la densidad, su porcentaje de variación fue $2,51 \%$, lo que indica una mayor influencia del aceite en la mezcla sobre la viscosidad en comparación con la densidad. También se realizó un análisis de correlación de Pearson, donde se comprobó que existe una relación muy fuerte casi perfecta con $\mathrm{R}$ $=0,99$ (Hopkins, 2014) y significativa ( $\mathrm{p}$-valor $<0,05)$ entre el porcentaje de aceite de Jatropha gossypiifolia en la disolución y la viscosidad e igualmente se obtuvo que también existe relación muy fuerte $(\mathrm{R}=0,93)$ y significativa ( $\mathrm{p}$-valor $<0,05)$ respecto a la densidad. Se demuestra que los porcentajes de aceite en las disoluciones influyen en las propiedades de las mismas.
En la tabla 6 se observa como el índice de inestabilidad de la muestra disminuye al aumentar la cantidad de aceite de Jatropha gossypiifolia en la disolución, lo que significa que se utiliza menor cantidad de dispersante, observándose un mejor desempeño de las disoluciones, respecto al dispersante patrón (xileno).

Tabla 6. Índices de inestabilidad obtenidos al aplicar las disoluciones preparadas.

\begin{tabular}{|l|c|c|}
\hline \multicolumn{1}{|c|}{ Dispersante } & INE a $\mathbf{2 5}^{\circ} \mathbf{C}$ & INE a $\mathbf{4 0}{ }^{\circ} \mathbf{C}$ \\
\hline Xileno & $0,38 \pm 0,02$ & $0,54 \pm 0,03$ \\
\hline A & $0,28 \pm 0,01$ & $0,29 \pm 0,02$ \\
\hline B & $0,08 \pm 0,01$ & $0,19 \pm 0,01$ \\
\hline C & $0,02 \pm 0,01$ & $0,03 \pm 0,01$ \\
\hline D & $0,01 \pm 0,005$ & $0,02 \pm 0,01$ \\
\hline E & $0,02 \pm 0,01$ & $0,03 \pm 0,01$ \\
\hline
\end{tabular}

Fuente: elaboración propia.

Un análisis de correlación muestra una correlación inversa muy fuerte $(\mathrm{R}=-0,92)$ y significativa ( $\mathrm{p}$-valor $<0,05$ ) entre el porcentaje de aceite de Jatropha gossypiifolia en la disolución y el valor de INE a ambas temperaturas (Hopkins, 2014) lo que demuestra que existe una asociación inversamente proporcional entre ambas variables. Se observa un mejor desempeño de las disoluciones a la temperatura de $25^{\circ} \mathrm{C}$ y a partir de $30 \%$ aceite (C).

Los resultados mostrados en la tabla 7, demuestran la eficiencia de las disoluciones preparadas, en donde se observa que para ambas temperaturas las disoluciones con 30,40 y $50 \%$ de aceite fueron las de mayor eficiencia con más de $94 \%$.

Tabla 7. Eficiencias calculadas para cada \%Aceite.

\begin{tabular}{|c|c|c|}
\hline \%Aceite & \%EF a $\mathbf{~ 2 5}^{\mathbf{}} \mathbf{C}$ & \%EF a $\mathbf{4 0}^{\mathbf{}} \mathbf{C}$ \\
\hline 10 & 25,0 & 46,3 \\
\hline 20 & 77,8 & 64,8 \\
\hline 30 & 94,4 & 94,4 \\
\hline 40 & 97,2 & 96,3 \\
\hline 50 & 95,8 & 95,4 \\
\hline
\end{tabular}

Fuente: elaboración propia.

Las eficiencias máximas se obtienen con la disolución de $50 \%$ aceite y $50 \%$ gasoil, siendo mayor para la temperatura de $25^{\circ} \mathrm{C}$ con un valor de $97,2 \%$. Este valor representa el porcentaje en que el tratamiento supera el desempeño del xileno como dispersante patrón. Un análisis estadístico descriptivo arrojó como resultado que los coeficientes de variación fueron mayores a $5 \%$, con $39,30 \%$ y $28,67 \%$ para 25 y $40{ }^{\circ} \mathrm{C}$ respectivamente, lo que indica que el parámetro eficiencia es dependiente de los porcentajes de aceite presentes en las disoluciones. 
El resultado del análisis de varianza multifactorial realizado se muestra en la tabla 8 , en la que se observa que de los dos factores experimentales, sólo el factor disolución resultó estadísticamente significativo por presentar un p-valor $<0,05$. El factor temperatura no influyó de forma significativa sobre los valores de eficiencia de dispersión ( $\mathrm{p}$-valor $>0,05$ ).

Tabla 8. Análisis de varianza multifactorial para IE.

\begin{tabular}{|l|c|c|c|}
\hline Factor & Suma cuadrados & GL & Valor-P \\
\hline Temperatura & 4,9 & 1 & 0,813 \\
\hline Disolución & 5531,91 & 4 & 0,008 \\
\hline
\end{tabular}

Fuente: elaboración propia.

Se puede decir entonces que para la muestra de petróleo crudo estudiada y bajo las condiciones de la investigación, la eficiencia de las disoluciones de aceite de Jatropha gossypiifolia en gasoil es independiente de la temperatura a la cual se caliente el petróleo, pero es dependiente del porcentaje de aceite presente en la mezcla.

En la tabla 9 se muestra el resultado del contraste múltiple de rangos aplicado a la variable eficiencia en función al factor Disolución, mediante el test HSD de Tukey, el cual se realizó para observar las diferencias individuales de los diferentes tratamientos.

Tabla 9. Contraste múltiple de rangos de Tukey (HSD) para eficiencia por Disolución.

\begin{tabular}{|c|c|c|c|c|c|}
\hline \%Aceite & Casos & Media & \multicolumn{2}{|c|}{ Grupo } & \multirow{2}{*}{ HSD } \\
\hline 10 & 2 & 35,7 & A & & \\
\hline 20 & 2 & 71,3 & A & B & \multirow{2}{*}{38,7} \\
\hline 30 & 2 & 94,4 & & B & \\
\hline 40 & 2 & 95,6 & & B & \\
\hline 50 & 2 & 96,8 & & B & \\
\hline
\end{tabular}

Fuente: elaboración propia.

En cuanto al contraste múltiple de rangos aplicado al factor Disolución cuyos resultados se muestran en la tabla 9, se observa que no existe diferencia estadísticamente significativa entre los resultados de eficiencia para las disoluciones con 30,40 y $50 \%$ de aceite en gasoil por ubicarse todas en el grupo B y que este grupo presenta diferencias significativas con las disoluciones que contienen 10 y $20 \%$ de aceite (grupos A y $A B)$.

Se comprueba entonces lo mostrado en el ANOVA multifactorial, el cual indicó el efecto del factor disolución y que a partir del 30\% la eficiencia de dispersión se mantiene constante, por lo que éste representa el porcentaje de aceite con mejor desempeño, pues es el menor porcentaje de aceite que presenta la mejor eficiencia de dispersión.

\section{Discusión}

Las propiedades de la muestra de petróleo utilizada para el estudio (tabla 2), corresponden en promedio a las producidas en el Campo El Furrial del Estado Monagas, Venezuela, lo que está en concordancia con lo que establece Beltrán, (2010). Aunque no se puede generalizar con la composición SARA del petróleo, la tendencia observada guarda cierto grado de similitud con los valores reportados por Lamus et al. (2011) correspondientes a análisis realizados a un petróleo crudo mediano de Colombia, mostrando valores de Saturados entre 27 y 54\%, Aromáticos entre 21 y 35\%, Resinas entre 5 y $11 \%$ y Asfaltenos entre 1 y $9 \%$. Se observó que los valores obtenidos en la presente investigación se encuentran en los rangos mostrados, a excepción de los aromáticos, sin embargo es de hacer notar que la composición del petróleo varía de una zona productora a otra, por lo que no se puede generalizar.

Al calcular los índices de estabilidad de los asfaltenos con base en la composición SARA, se obtuvo que el mismo presenta características inestables, lo que implica que tienen tendencia a producir depósitos orgánicos durante su producción, tratamiento y procesamiento. Esta observación coincide con lo analizado por Carbognani, Méndez y Sánchez (1999) quienes sostienen, sobre la base de resultados de análisis composicional y pruebas de campo, que la mayoría de los petróleos producidos en Venezuela presentan características inestables, por su alta relación Saturados/asfaltenos. Igualmente Ashoori et al. (2016) al investigar sobre la relación entre la composición SARA y la estabilidad de petróleos de Irán, concluyeron que la estabilidad es función de la relación entre todos los componentes del petróleo y no de relaciones individuales, lo que es un indicativo de la importancia de la misma para caracterizar la tendencia a la estabilidad.

Al analizar las propiedades del aceite de Jatropha gossypiifolia obtenidas (tabla 3), se observó un rendimiento respecto al total extraído de $27 \%$, que al ser comparado con resultados reportados por otras investigaciones, se encuentra entre $23,9 \%$ (De Oliveira et al., 2009) y 28,5\% (Banerji et al., 1985), lo que indica que la materia prima utilizada tiene un rendimiento acorde y aceptable, aunque otros autores refieren que la semilla contiene hasta un $40 \%$ de aceite (Mazza, Puccio \& Franke, 2017) y 32,0\% (Hosamani $\&$ Katagi, 2008). Entre los estudios realizados al aceite 
de Jatropha gossypiifolia, se encuentra el de Oliveira et al. (2009) quienes consideraron que el aceite de esta especie tiene potencial para la elaboración de biodiesel, además reporta un porcentaje de rendimiento másico de aceite de $23,9 \%$ valor menor al obtenido en la presente investigación, lo que demuestra que en comparación las semillas utilizadas tuvieron un buen rendimiento de aceite.

De Oliveira et al. (2009) también reportan una densidad del aceite de $0,92 \mathrm{~g} / \mathrm{mL}$, lo que representa un mayor valor en comparación con el obtenido $(0,88 \mathrm{~g} / \mathrm{mL})$, aun cuando se utilizó el mismo solvente, es claro que la diferencia se puede deber a que se utilizaron semillas de plantas cultivadas en diferentes lugares, por lo que se debe profundizar en la influencia del clima, suelo y condiciones de crecimiento. Al ser un aceite poco estudiado, no se tiene referencia de otras propiedades como la viscosidad, ya que los estudios se han basado principalmente en la composición del aceite y sus posibles usos medicinales (Okoh et al., 2016; Falodun et al., 2012).

El análisis de la insolubilidad (UF) y solubilidad (PD) de la muestra de petróleo crudo original sin tratamiento mostró una potencial dependencia de la estabilidad con la temperatura, partiendo del principio tomado como referencia en la investigación de que a menor INE mayor es la estabilidad. Al calcular el INE para el ensayo realizado a $25{ }^{\circ} \mathrm{C}$ se observó un menor valor que al realizarlo a $40{ }^{\circ} \mathrm{C}$, obteniéndose un aumento del INE en $33,7 \%$. El efecto de la temperatura sobre la estabilidad de los asfaltenos ha sido motivo de debate por parte de los investigadores, uno de los primeros trabajos realizados fue el de Hirschberg et al. (1984) quienes en pruebas realizadas a nivel de laboratorio mostraron una relación inversa entre la temperatura y la solubilidad de los asfaltenos en el petróleo. La investigación realizada por Mohammadi et al. (2016) sobre el efecto de la temperatura en la agregación de asfaltenos en un petróleo crudo liviano de Irán, concluyen que el aumento de ésta al generar la disminución de la viscosidad del petróleo produce un aumento en la difusividad efectiva y la frecuencia de colisión de las partículas de asfaltenos, produciendo a su vez un aumento en la velocidad de formación y crecimiento de los agregados. Más recientemente, Torkaman, Bahrami y Dehghani (2017) reportaron que en petróleos crudos del golfo pérsico la estabilidad de los asfaltenos aumenta con la temperatura, es decir un resultado contrario al obtenido en la presente investigación.
El comportamiento del INE al aplicar cada una de las disoluciones dispersantes de aceite de Jatropha gossypiifolia en gasoil (tabla 6), muestra como independientemente de la disolución y de la temperatura, todas son capaces de estabilizar los asfaltenos, ya que se utiliza menor cantidad de las mismas, respecto a la cantidad de xileno aplicado originalmente, lo que induce a que el INE sea menor. Se demuestra la aplicabilidad del aceite como componente activo para la dispersión de agregados de asfaltenos, lo que concuerda con lo obtenido por Marín, Marcano y Febres (2016) al utilizar aceite de otra planta del género, la Jatropha curcas, demostrándose el potencial de estos aceites para ser utilizados en la prevención y corrección de los problemas de precipitación de asfaltenos. En otra investigación Bello et al. (2015) concluyeron que otro aceite vegetal, el de Cocos nucifera, también tiene efectos positivos en la dispersión de asfaltenos y estos aumentan al aumentar la concentración del mismo en gasoil. Rocha, Silva y Da Silva (2006) también estudiaron la capacidad dispersante de asfaltenos de varios aceites vegetales aplicados a petróleos crudos brasileños: pino, coco, pimienta jamaicana, nuez de Brasil, semilla de uva, almendra dulce, andiroba, y sándalo. En el estudio citado anteriormente se corroboró que todos los aceites vegetales mostraron capacidad dispersora, destacándose los de coco, avellana y sándalo. Se puede decir entonces que los aceites vegetales y sobre todo el de Jatropha gossypiifolia tienen un gran potencial para ser utilizados en la industria petrolera, más aun cuando se trata de una especie que produce un aceite no comestible y tóxico, lo que es una ventaja respecto a otros aceites como el de coco y avellanas.

Según lo reportó De Oliveira et al. (2009) el aceite de Jatropha gossypiifolia está compuesto mayormente por ácido palmítico y ácido oleico. Estos ácidos tiene como grupo funcional activo el carboxílico, el cual le puede aportar características de surfactante, lo que puede influir directamente en su efecto estabilizante de asfaltenos, aunque se debe confirmar esta observación realizando estudios químicos más específicos.

Con la aplicación de las disoluciones de aceite en gasoil, se obtuvo una eficiencia máxima respecto al INE original con xileno de $97,2 \%$ (tabla 7), que supera a las reportadas para el aceite de Jatropha curcas (Marín et al., 2016) el cual fue de $88,33 \%$ máximo. El resultado obtenido también es superior al reportado por Pereira et al. (2011), quienes determinaron para un producto dispersante comercial una eficiencia de 73,5\%. En su investigación Atta et al. (2017) al evaluar un dispersante 
formulado con base en un líquido iónico, reportaron una eficiencia máxima de 59,19\%, valor también bajo respecto al máximo obtenido en la presente investigación. Al igual que lo observado al analizar los INE, los valores de eficiencia demuestran que la mezcla entre el aceite de Jatropha gossypiifolia y gasoil es más eficiente para la dispersión de los agregados de asfaltenos en comparación con otros productos tanto vegetales como sintéticos, por lo que se debe evaluar su posible uso para remover o prevenir depósitos orgánicos a nivel de campo.

El análisis estadístico realizado demostró que de los dos factores experimentales evaluados, solo las disoluciones tiene efecto significativo con un nivel de confianza de $95 \%$ (tabla 8). La temperatura en el rango de investigación, no afectó el desempeño de las disoluciones bajo las condiciones de los ensayos realizados, por lo tanto se descarta un efecto de este parámetro sobre la eficiencia del aceite al ser utilizado como dispersante de asfaltenos. Lo anterior indica que a pesar de las diferencias numéricas observadas al aplicar las diferentes disoluciones a las dos temperaturas, éstas no fueron estadísticamente significativas. El efecto de la temperatura de ensayo para la determinación de la eficiencia de los dispersantes de asfaltenos ha sido poco estudiada, ya que los diferentes métodos se basan en temperatura preestablecidas. La mayoría de los trabajos, como el de Barcenas et al. (2008) se basan en la determinación de la eficiencia de diferentes dispersantes bajo las mismas condiciones, no en la eficiencia de uno en particular a condiciones diferentes de temperatura.

El análisis de HSD de Tukey aplicado al factor disolución mostró que a pesar de que se tienen diferencias significativas en el análisis ANOVA, a partir de la disolución con 30\% aceite de Jatropha gossypiifolia, la eficiencia se mantiene constante con un nivel de significancia de 0,05 . Este resultado coincide con el obtenido para el aceite de Jatropha curcas (Marín et al., 2016) donde se reportó que no se obtuvieron diferencias significativas entre las eficiencias para concentraciones de 20,40 y $60 \%$ de aceite en gasoil. Este resultado sugiere que ambos aceites se comportan de forma similar, lo que se puede deber a sus composiciones. Los aceites de ambas plantas poseen composición química similar, según lo mostrado por Cardoso et al. (2012) con proporciones mayoritarias de ácido oleico mono insaturado $(18: 1 ; 34,3$ - 45,8\%) y ácido linoleico di insaturado (18:2; 29,0 - 44,2\%). Así mismo, Aboaba et al. (2015) reportó que la composición del aceite de Jatropha gossypiifolia presenta una cantidad importante de hidrocarburos terpénicos, entre los que destacan los sesquiperpenos oxigenados, los cuales pueden actuar como surfactantes, de allí su eficiencia en la dispersión de los asfaltenos. Resultados de composición del aceite similares fueron reportados también por Moreti et al. (2013) con una prevalencia de los ácidos linoleico (24,09\%) y heptadecanóico $(35,81 \%)$.

\section{Conclusiones y recomendaciones}

Se concluye que bajo las condiciones de la investigación y de acuerdo al diseño experimental planteado, al aumentar el porcentaje de aceite de Jatropha gossypiifolia en la disolución con gasoil, disminuye en Índice de inestabilidad en la muestra de petróleo crudo utilizada, lo que es indicativo de su efecto estabilizante sobre los asfaltenos.

Estadísticamente se demostró que el efecto estabilizante de las disoluciones de aceite de Jatropha gossypiifolia en gasoil se ve afectado por el porcentaje de aceite, pero es independiente de la temperatura en el intervalo en el que se realizaron los ensayos, con un nivel de confianza de $95 \%$, sin embargo es recomendable estudiar el efecto de la temperatura en un rango más amplio para observar la influencia de esta variable de manera más profunda.

Se obtuvo que las diferencias en la eficiencia de las disoluciones de aceite de Jatropha gossypiifolia en gasoil como dispersantes de asfaltenos no fue estadísticamente significativa para porcentajes de 30,40 y $50 \%$ con valores mayores a $94 \%$, aunque la máxima eficiencia se obtuvo con la disolución al $40 \%$ aceite con la que se obtuvo valores de 97,2 y $96,3 \%$ a las temperaturas de 25 y $40{ }^{\circ} \mathrm{C}$ respectivamente.

Se demostró la factibilidad de utilizar el aceite de la Jatropha gossypiifolia como agente activo disuelto en gasoil para la dispersión de los asfaltenos en un petróleo crudo mediano con características inestables, según sus valores de relación saturados/asfaltenos y su índice de estabilidad coloidal, por lo que se recomienda continuar esta línea de investigación utilizando otros solventes y otras condiciones operacionales como la presión, así mismo realizar ensayos con otros tipos de petróleos crudos y en condiciones dinámicas.

\section{Referencias}

Aboaba, S.A., Adebayo, M.A., Ogunwande, I.A., \& Olayiwola, T.O. (2015). Volatile constituents of Jatropha gossypifolia L. grown in Nigeria. American Journal of Essential Oils and Natural Products, 2(4), 08-11.

Afra, S., Nasr-El-Din, H.A., Socci, D., \& Cui, Z. (2018). Green phenolic amphiphile as a viscosity modifier and asphaltenes dispersant for heavy 
and extra-heavy oil. Fuel, 220, 481-489. doi: 10.1016/j.fuel.2018.01.111

Akbarzadeh, K., Allenson, S., Creek, J., \& Jamaluddin, A. (2007). Asphaltenes-Problematic but Rich in Potential. Oilfield Review, 9(2), 22-48.

Alayón, M. (2004). Asfaltenos. Ocurrencia y floculación. Mérida, Venezuela: Universidad de Los Andes.

Andersen, S.I. (1999). Flocculation Onset Titration of Petroleum Asphaltenes. Energy \& Fuel, 13, 315-322.

Ashoori, S., Sharifi, M., Masoumi, M., \& Salehi, M.M. (2017). The relationship between SARA fractions and crude oil stability. Egyptian Journal of Petroleum, 26, 209-213.

Asomaning, S., \& Watkinson, A.P. (2000). Petroleum Stability and Heteroatom Species Effects in Fouling of Heat Exchangers by Asphaltenes. Heat Transfer Engineering, 21(3), 10-16. doi: 10.1080/014576300270852.

Atta, A.M., Ezzat, A.O., Abdullah, M.M., \& Hashem, A.I. (2017). Effect of Different Families of Hydrophobic Anions of Imadazolium Ionic Liquids on Asphaltene Dispersants in Heavy Crude Oil. Energy Fuels, 31, 8045-8053. doi: 10.1021/acs.energyfuels. $7 \mathrm{~b} 01167$.

ASTM D287. (2012). Standard Test Method for API Gravity of Crude Petroleum and Petroleum Products (Hydrometer Method). West Conshohocken, PA: American Society for Testing and Materials.

ASTM D445. (2011). Standard Test Method for Kinematic Viscosity of Transparent and Opaque Liquids (and Calculation of Dynamic Viscosity). West Conshohocken, PA: American Society for Testing and Materials.

ASTM D891. (2009). Standard Test Methods for Specific Gravity, Apparent, of Liquid Industrial Chemicals. West Conshohocken, PA: American Society for Testing and Materials.

ASTM D2007. (2011). Standard Test Method for Characteristic Groups in Rubber Extender and Processing Oils and Other Petroleum-Derived Oils by the Clay-Gel Absorption Chromatographic Method. West Conshohocken, PA: American Society for Testing and Materials.

ASTM D2196. (2010). Standard Test Methods for Rheological Properties of Non-Newtonian Materials by Rotational Viscometer. West Conshohocken, PA: American Society for Testing and Materials.

Banerji, R., Chowdhury, A., Misra, G., Sudarsanam, G., Verma, S., \& Srivastava, G. (1985). Jatropha
Seed Oils for Energy. Biomass, 8(1), 277-282.

Barcenas, M., Orea, P., Buenrostro-González, E., Zamudio-Rivera, L.S., \& Duda, Y. (2008). Study of Medium Effect on Asphaltene Agglomeration Inhibitor Efficiency. Energy \& Fuels, 22(3), 1917-1922. doi: 10.1021/ ef700773m.

Bello, Y.B., Manzano, J.R., \& Marín, T.D. (2015). Análisis comparativo de la eficiencia dispersora de asfaltenos de productos a base de aceite de coco (Cocus nucifera) como componente activo y dispersantes comerciales aplicados a muestras de petróleo del Campo el Furrial, Estado Monagas, Venezuela. Revista Tecnológica ESPOL - RTE, 28(2), 51-61.

Beltrán, O.J. (2010). Caracterización petrofísica y sedimentológica mediante el análisis de núcleos en yacimientos de la formación Naricual, cuenca oriental de Venezuela (tesis de maestría). Universidad del Zulia, Venezuela.

Campen, S., Moorhouse, S.J., \& Wong, J.S.S. (2019). Mechanism of an asphaltene inhibitor in different depositing environments: Influence of colloid stability. Journal of Petroleum Science and Engineering, 106502. doi: 10.1016/j. petrol.2019.106502.

Carbognani, L., Méndez, A., \& Sánchez, V. (1999). Caracterización de crudos venezolanos. Orígenes, razones y necesidades. Acta Científica Venezolana, 50(1), 11-17.

Cardoso, K.C., Da Silva, M.J., Grimaldi, R., Stahl, M., Simas, R.C., Cunha, I.B.S., Eberlin, M.N., \& Alberici, R.M. (2011). TAG Profiles of Jatropha curcas L. Seed Oil by Easy Ambient SonicSpray Ionization Mass Spectrometry. Journal of the American Oil Chemists' Society, 89(1), 6771. doi: 10.1007/s11746-011-1888-7.

Chavez, A. C. R., Campo, L. T. C., Morales, A. R., \& Carrillo, Z. C. (2011). Asfaltenos, un daño severo en yacimientos altamente subsaturados de aceite volátil. Revista Fuentes, 9(2).

Cuffaro, K.A., \& Centeno, L.P. (2017). Comparación de la eficiencia de los aceites de piñón (Jatropha curcas) e higuereta (Ricinus communis) como dispersantes de asfaltenos aplicados a un crudo mediano del norte de Monagas (tesis de pregrado). Universidad de Oriente, Venezuela.

De Oliveira, J.S., Leite, P.M., De Souza, L.B., Mello, V.M., Silva, E.C., Rubim, J.C., Meneghetti, S.M.P., \& Suarez, P.A.Z. (2009). Characteristics and composition of Jatropha gossypiifolia and Jatropha curcas L. oils and application for biodiesel production. Biomass and bioenergy, 
33, 449-453.

De Almeida, P.M., De Sousa, S., Marin-Morales, M.A., Benko-Iseppon, A.M., \& Brasileiro-Vidal, A.C. (2015). Genotoxic potential of the latex from cotton-leaf physicnut (Jatropha gossypiifolia L.). Genetics and Molecular Biology, 38(1), 93-100.

De Almeida, P.M., De Sousa, S.S., Santos, I.R.M.R., Marin-Morales, M.A., Benko-Iseppon, A.M., Santos, A.V., Randau, K.P., \& BrasileiroVidal, A.C. (2016). Genotoxic potential of leaf extracts of Jatropha gossypiifolia L. Genetics and Molecular Research, 15(1), 1-9. doi: 10.4238/gmr.15017415.

Falodun, A., Sheng-Xiang, Q., Parkinson, G., \& Gibbons, S. (2012). Isolation and characterization of a new anticancer diterpenoid from Jatropha gossypifolia. Pharmaceutical Chemistry Journal, 45(10), 636-639. doi: 10.1007/s11094012-0693-4.

Félix-Silva, J., Giordani, R.B., Da Silva-Jr, A.A., Zucolotto, S.M., \& Fernandes-Pedrosa, M. (2014). Jatropha gossypiifolia L. (Euphorbiaceae): A Review of Traditional Uses, Phytochemistry, Pharmacology, and Toxicology of This Medicinal Plant. EvidenceBased Complementary and Alternative Medicine, 1-32. doi: 10.1155/2014/369204.

Figueira, J. N., Simão, R. A., Soares, B. G., \& Lucas, E. F. (2017). The influence of chemicals on asphaltenes precipitation: a comparison between atomic force microscopy and near infrared techniques. Revista Fuentes, 15(1), 7-17.

García, C. A., Rodríguez, O. C., Casallas, P. A., Cruz, G., Hernández, F. E., Afanador, L. E., \& Rodríguez, L. (2010). Optimización del transporte por oleoducto de crudo pesado Castilla. Fuentes: El reventón energético, 8(1), 2.

Ghaffar, A.M.A., Kabel, K.I., Farag, R.K., Maysour, N.E., \& Zahran, M.A.H. (2013). Synthesis of poly (dodecyl phenol formaldehyde)b-poly (oxypropylene) block copolymer, and evaluation as asphaltene inhibitor and dispersant. Research on Chemical Intermediates, 41(1), 443-455. doi: 10.1007/ s11164-013-1243-8.

Goual, L. (2012). Petroleum Asphaltenes. USA: University of Wyoming.

Hirschberg, A., Dejong, L.N.J., Shipper, B.A., Meijer, J.G. (1984). Influence of temperatura and pressure on asphaltene flocculation. Society of Petroleum Engineers Journal, 24(3), 283-293. doi: 10.2118/11202-pa.
Hopkins, W. (2014). A new view of statistics. Recuperado de https://complementarytraining.net/free-willhopkins-a-new-view-of-statistics-pdf-printout/.

Hosamani, K.M., \& Katagi, K.S. (2008). Characterization and structure elucidation of 12-hydroxyoctadec-cis-9-enoic acid in Jatropha gossypifolia and Hevea brasiliensis seed oils: a rich source of hydroxy fatty acid. Chemistry and Physics of Lipids, 152, 9-12.

Islam, M., Islam, A., \& Hossain, M.S. (2017). Biological Investigation of Jatropha gossypiifolia: A Stiff Medicinal Plant in Bangladesh. Iranian Journal of Pharmaceutical Sciences, 13(1), 35-48.

Kumar, A., \& Singh, N. (2012). Jatropha gossypiifolia L.: a potential genetic resource for herbal dye. Genetic Resources and Crop Evolution, 59(5), 949-954. doi: 10.1007/s10722-012-9815-9.

Lamus, C., Guzmán, A., Murcia, B., Cabanzo, R., \& Mejía-Ospino, E. (2011). Uso de Análisis Multivariado En La Determinación SARA De Crudos Por Espectroscopia NIR. Revista Colombiana de Física, 43(3), 635-642.

León, E. A. (2011). De la caracterización de crudos qué es clave para diagnosticar la precipitación de parafinas. Fuentes: El reventón energético, 9(1), 4.

León, E. A., González, F. E. C., \& Arango, J. F. Z. (2007). Estudio comparativo de los modelos de precipitación de parafinas. Fuentes: El reventón energético, 5(1), 2.

León, E. A., Quintero, J. F. G., Cervantes, A. A. B., \& Rangel, E. R. S. (2012). Análisis de la simulación de precipitación de asfaltenos en el crudo del campo Colorado. Fuentes: El reventón energético, 10(1), 6.

Lim, S.H., Go, K.S., Nho, N.S., \& Lee, J.G. (2018). Effect of reaction temperature and time on the products and asphaltene dispersion stability in slurry-phase hydrocracking of vacuum residue. Fuel, 234, 305-311. doi: 10.1016/j. fuel.2018.06.113.

Mazza, G., Puccio, P., \& Franke, S. (2017). Jatropha gossypiifolia. Recuperado de http://www.photomazza.com/? Jatrophagossypiifolia\&lang=es.

Madh, M., Kharrat, R., \& Hamoule, T. (2017). Screening of inhibitors for remediation of asphaltene deposits: Experimental and modeling study. Petroleum. doi: 10.1016/j.petlm.2017.08.001.

Mansoori, G. (2009). A unified perspective on the phase behaviour of petroleum fluids. International Journal Oil, Gas and Coal Technology, 2(2), 141-167. 
Maqbool, T., Srikiratiwong, P., \& Fogler, H.S. (2011). Effect of Temperature on the Precipitation Kinetics of Asphaltenes. Energy Fuels, 25, 694-700. doi:10.1021/ef101112r.

Marín, T., Marcano, S., \& Febres, M. (2016). Evaluación del aceite de Jatropha curcas como aditivo dispersante de asfaltenos en un crudo del campo el Furrial, Venezuela. Revista Ingeniería, 20(2), 99-107.

Mohammadi, S., Rashidi, F., Mousavi-Dehghani, S.A., Ghazanfari, M.H. (2016). On the effect of temperature on precipitation and aggregation of asphaltenes in light live oils. The Canadian Journal of Chemical Engineering, 94(9), 18201829. doi: 10.1002/cjce.22555.

Moreti, J., Ferreira, W., Minguzzi, S., De Lara, R.C. (2013). Assessment of chemical composition and toxicity of the essential oil ofleaves and fruits of Jatropha gossypifolia L. Semina: Ciências Exatas e Tecnológicas, Londrina, 34(2), 185192. doi: 10.5433/1679-0375.2013v34n2p185.

Okoh, S.O., Iweriebor, B.C., Okoh, O.O., \& Okoh, A.I. (2016). Antibacterial and Antioxidant Properties of the Leaves and Stem Essential Oils of Jatropha gossypifolia L. BioMed Research International, 1-9. doi: 10.1155/2016/9392716.

Oyedeji, K.O., Akinbiyi, P., Oni, J., \& Obisesan, A. (2018). Visceral Histopathological Alterations in Rats Treated with Ethanol Extract of Jatropha Gossypifolia (POHL). Journal of Pharmaceutical Science and Research, 10(5), 1027-1029.

Pereira, J.C., Delgado-Linares, J., Briones, A., Guevara, M., Scorzza, C., \& Salager, J.L. (2011). The Effect of Solvent Nature and Dispersant Performance on Asphaltene Precipitation from Diluted Solutions of Instable Crude Oil. Petroleum Science and Technology, 29, 2432-2440.

Pineda-Flores, G., \& Mesta-Howard, A. (2001). Petroleum asphaltenes: generated problematic and possible biodegradation mechanisms. Revista Latinoamericana de Microbiología, 43(3), 143-150.

Rezaee, E., Heidarizadeh, F., Sajjadifar, S., \& Abbasi, Z. (2013). Dispersing of Petroleum Asphaltenes by Acidic Ionic Liquid and Determination by UV-Visible Spectroscopy. Journal of Petroleum Engineering, 1-5. doi: 10.1155/2013/203036.

Rocha, L.C., Silva, M., \& Da Silva, A.C. (2006). Inhibition of asphaltene precipitation in Brazilian crude oils using new oil soluble amphiphiles. Journal of Petroleum Science and Engineering, 51, 26-36. doi: 10.1016/j. petrol.2005.11.006.

Romero, J.F., Feitosa, F.X., Do Carmo, F.R., \& De Sant'Ana, H.B. (2018). Paraffin effects on the stability and precipitation of crude oil asphaltenes: Experimental onset determination and phase behavior approach. Fluid Phase Equilibria, 474, 116-125. doi: 10.1016/j. fluid.2018.07.017.

Saeedi, A.H., \& Hasan, M. (2017). Inhibiting asphaltene precipitation from Iranian crude oil using various dispersants: Experimental investigation through viscometry and thermodynamic modelling. Fluid Phase Equilibria, 442, 104-118.

Safaie, K., \& Naza, A. (2014). Evaluation of Asphaltene Inhibitors Effect on Aggregation Coupled Sedimentation Process. Journal of Dispersion Science and Technology, 35(3), 329-337.

Salazar, D., Rodríguez, S., \& Marín, T. (2017). Estudio de las propiedades fisicoquímicas de un biodiesel elaborado a partir del aceite de semillas de piñón criollo (Jatropha curcas L.). Revista Científica PetroCiencias, 1(1), 48-61.

Sanchez, A. F. P., Sanchez, J. D. B., Gonzalez, J. F. R., \& Ramirez, L. E. M. (2017). Transporte de crudo pesado por oleoducto usando el método de dilución: Un enfoque práctico para modelar la caída de presión y la precipitación de asfaltenos. Revista Fuentes, 15(2), 7-17.

Spiecker, P.M., Gawrys, K.L., \& Kilpatrick, P.K. (2003). Aggregation and solubility behavior of asphaltenes and their subfractions. Journal of Colloid and Interface Science, 267, 178-193.

Sepúlveda, J., Bonilla, J., \& Medina, Y. (2010). Predicción de la Estabilidad de los Asfaltenos Mediante la Utilización del Análisis SARA para Petróleos Puros. Revista Ingeniería y Región, 7(1), 103-110.

Sulaimon, A.A., De Castro, J.K.M., \& Vatsa, S. (2019). New correlations and deposition envelopes for predicting asphaltene stability in crude oils. Journal of Petroleum Science and Engineering, 106782. doi: 10.1016/j.petrol.2019.106782.

Torkaman, M., Bahrami, M., Dehghani, M. (2017). Influence of Temperature on Aggregation and Stability of Asphaltenes. I. Perikinetic Aggregation. Energy Fuels, 31, 11169-11180. doi: 10.1021/acs.energyfuels.7b00417.

Wu, Q., Patocka, J., Nepovimova, E., \& Kuca, K. (2019). Jatropha gossypiifolia L. and its biologically active metabolites: A mini review. Journal of Ethnopharmacology, 234, 197-203. 
Xavier-Santos, J.B., Félix-Silva, J., Passosa, J.C.R., Gomes, J.A.S., Fernandes, J., Barreto-Garcia, V., De Araujo-Junior, R., Zucolottob, S.M., Silva-Junior, A.A., \& Fernandes-Pedrosa, M.F. (2018). Development of an effective and safe topical anti-inflammatory gel containing Jatropha gossypiifolia leaf extract: Results from a pre-clinical trial in mice. Journal of Ethnopharmacology, 227, 268-278. doi: 10.1016/j.jep.2018.09.007.

Recepción: 18 de mayo de 2019

Aceptación: 24 de febrero de 2020 